\title{
Conocimiento y prácticas sobre la lactancia materna exclusiva en madres primíparas que acuden a consultar a dos hospitales amigo del niño de Paraguay
}

\author{
Knowledge and practices on exclusive breastfeeding in primiparous \\ mothers who consult in two child friendly hospitals in Paraguay
}

\begin{abstract}
Eliana Romina Meza Miranda*1 ${ }^{\odot}$, Rubén Carlos Servín Salinas ${ }^{2}{ }^{\bullet}$, Liz Stefanie Borda Saldívar ${ }^{2}{ }^{1}$ Universidad Nacional de Asunción. Centro Multidisciplinario de Investigaciones Tecnológicas. San Lorenzo, Paraguay. ${ }^{2}$ Universidad Nacional de Asunción. Facultad de Ciencias Químicas. San Lorenzo, Paraguay. *Autor de correspondencia: eliana_romina@hotmail.es.
\end{abstract}

Recibido: 2 mayo 2021

Aceptado: 30 junio 2021

Recibido en versión modificada: 14 junio 2021

Meza Miranda, E. R., Servín Salinas, R. C. \& Borda Saldívar, L. S. (2021). Conocimiento y prácticas sobre lactancia materna exclusiva en madres primíparas que acuden a consultar a dos hospitales amigo del niño de Paraguay. Investigaciones y Estudios - UNA, 12(1), 27-33. https://doi.org/10.47133/IEUNA2113

Resumen. La lactancia materna brinda un medio incomparable para otorgar un alimento ideal para el crecimiento y desarrollo del recién nacido. El objetivo fue evaluar los conocimientos y las prácticas relacionadas a la lactancia materna exclusiva en madres primíparas que acuden a los hospitales Materno Infantil Reina Sofía de la Cruz Roja Paraguaya y Hospital Materno Infantil de Trinidad que tienen la denominación "amigo del niño y de la madre" y la asociación entre estas variables. Se realizó un estudio descriptivo analítico de corte transversal, realizado en los meses de enero y febrero del 2020 a 90 primíparas. Las variables estudiadas fueron sociodemográficas, de conocimientos y prácticas sobre lactancia materna exclusiva. La edad promedio fue de 27 . La mayoría eran solteras (46\%).
El secundario fue el nivel de escolaridad en general con un $49 \%$. La ocupación de las madres fue el de ama de casa en su mayoría (57 \%). En cuanto al conocimiento sobre lactancia materna exclusiva se encontraron las siguientes proporciones: regular fue el mayor con $53 \%$, seguido de bueno en un $29 \%$ y por último deficiente con $18 \%$. En cuanto al nivel de prácticas, se halló un $54 \%$ inadecuada y adecuada en 45\%. Al analizar si existe asociación entre los conocimientos y prácticas sobre lactancia materna exclusiva, no hemos encontrado asociación entre estas variables $(\mathrm{P}=0,233)$. El conocimiento sobre lactancia materna en primíparas fue regular en su mayoría, y en cuanto a las prácticas se observó un nivel inadecuado en mayor cantidad. No se encontró asociación entre el conocimiento y las prácticas sobre lactancia materna exclusiva.

Palabras clave. lactancia materna, conocimiento, prácticas, primíparas. 
Abstract. Breastfeeding provides an incomparable means of providing an ideal food for the growth and development of the newborn. The Objective to evaluate the knowledge and practices related to exclusive breastfeeding in first-time mothers who attend the Reina Sofía Maternal and Child Hospital of the Paraguayan Red Cross and the Trinidad Maternal and Child Hospital, which have the name "friend of the child and mother" and the association between these variables. A descriptive analytical crosssectional study was carried out, carried out in January and February 2020 on 90 primiparous women. The variables studied were sociodemographic, knowledge and practice about exclusive breastfeeding. The average age was 27 . The majority were single (46\%). Secondary was the level of education in general with $49 \%$. The mothers' occupation was that of a housewife for the most part $(57 \%)$. Regarding knowledge about exclusive breastfeeding, the following proportions were found: regular was the highest with $53 \%$, followed by good in $29 \%$ and finally deficient with $18 \%$. Regarding the level of practices, it was found to be $54 \%$ inadequate and $45 \%$ adequate. When analyzing whether there is an association between knowledge and practices on exclusive breastfeeding, we have not found an association between these variables $(P=0.233)$. Knowledge about breastfeeding in primiparous women was mostly regular, and in terms of practices, an inadequate level was observed in a greater quantity. No association was found between knowledge and practices about exclusive breastfeeding.

Keywords. breastfeeding, knowledge, practices, primiparous.

\section{INTRODUCCIÓN}

La lactancia materna brinda un medio incomparable para otorgar un alimento ideal para el crecimiento y desarrollo del recién nacido. La Organización Mundial de la Salud (OMS) recomienda que la lactancia sea exclusiva durante los primeros 6 meses de vida y que constituya parte importante de la alimentación durante el resto del primer año, si es posible continuar hasta los dos años de vida (Ilabaca \& Atalah, 2002).

Las madres primíparas presentan mayor negligencia y están en desventaja social con respecto a la lactancia materna. La idealización de la lactancia, la incertidumbre ante las dificultades como por ejemplo el dolor y las grietas en los pezones, la duración de cada toma o la dedicación que requiere, el deseo de privacidad durante las tomas, la responsabilidad compartida con el niño para lograr el éxito, y finalmente, la desorganización en sus vidas y la modificación del rol de la mujer ponen a las mismas en un nuevo desafío (Belintxon-Martín et al., 2011).

A nivel mundial, no más del $35 \%$ de los lactantes de todo el mundo reciben exclusivamente leche materna siquiera durante los cuatro primeros meses de vida y a menudo la alimentación complementaria empieza demasiado pronto o demasiado tarde, y en muchos casos los alimentos son nutricionalmente inadecuados e insalubres (Organización Mundial de la Salud, 2004).

Como parte de los esfuerzos de medición de la “Iniciación y Duración de la Lactancia Materna” se han realizado dos encuestas nacionales en los años 2004 y 2008 por el Centro Paraguayo de Estudios de Población (CEPEP, 2005). Los resultados de las 
encuestas muestran que en el Paraguay el 95,3 \% a nivel nacional amamantaron a sus hijos durante algún periodo, sin embargo, este porcentaje disminuye súbitamente a tan solo $8,5 \%$ entre los niños de 3 a 5 meses, además de una introducción precoz de la alimentación sólida, no coincidiendo con las recomendaciones a nivel mundial, regional o país (CEPEP, 2005).

El Instituto Nacional de Alimentación y Nutrición (INAN) con su libro de Las Guías Alimentarias del Paraguay (Instituto Nacional de Alimentación y Nutrición, 2020), y el Programa Nacional de Lactancia Materna que realiza diversas publicaciones para promover la Lactancia Materna Exclusiva (LME), proporcionan materiales útiles en educación sanitaria referida a la lactancia materna.

La iniciativa Hospitales Amigos del Niño y de la Madre (IHANM) es una estrategia sólida para promover la lactancia y ha logrado un cambio en la rutina hospitalaria, en cuanto al alojamiento conjunto, apego precoz y aumento de la prevalencia de la lactancia materna exclusiva al egreso hospitalario (Sanabria et al., 2005; Rosa et al., 2012).

Todas las estrategias de promoción llevan a un mismo fin, lograr una duración de lactancia mayor. Según una encuesta realizada a madres en periodo post parto temprano, las mujeres expresaron claramente su necesidad de recibir información y orientación sobre la lactancia y cómo cuidar bien al recién nacido, de tener comprensión y apoyo de parte del personal de salud con sus dudas y equivocaciones, y de sentirse acompañadas y apoyadas por sus parejas y familiares cercanos (Vásquez Munive et al., 2012).

Por más sencilla que pudiera ser, la decisión de amamantar es muy personal y de suma trascendencia; sin duda, de las primeras que deberá tomar la madre. Esta acción se dificulta cuando no se tiene la información suficiente, cuando la publicidad de fórmulas lácteas infantiles es más fuerte que la propia promoción de la Lactancia Materna y aún más cuando el ambiente social dificulta la práctica (Rosa et al., 2012).

Por todo lo mencionado anteriormente, el presente trabajo tiene como objetivo evaluar los conocimientos y prácticas sobre lactancia materna exclusiva en las madres primíparas y determinar si existe asociación entre estas variables.

\section{MATERIALES Y MÉTODOS}

Diseño: estudio descriptivo analítico de corte transverso. El proceso de muestreo fue no probabilístico por conveniencia. Se incluyeron 90 madres primíparas, de las cuales 50 acudieron a consultar en el hospital Reina Sofía de la Cruz Roja Paraguaya y 40 al hospital Materno Infantil de Trinidad entre los meses de enero y febrero del año 2020, que manifestaron su deseo de participación y dieron su consentimiento informado. El presente estudio fue aprobado por el Comité de Ética de la Facultad de Ciencias Químicas de la Universidad Nacional de Asunción de Paraguay y se llevó a cabo con financiación propia. Los autores declaran no tener conflictos de intereses.

Las variables de estudio fueron: edad, estado civil, nivel de escolaridad, ocupación, conocimientos y prácticas de lactancia materna exclusiva utilizando el cuestionario adaptado por el autor Carranza (2016), la misma cuenta de tres partes, la primera referente a los datos sociodemográficos y estilo de vida, la segunda relacionada a los conocimientos sobre la lactancia materna y la tercera a las prácticas sobre lactancia materna. La misma cuenta con preguntas abiertas y cerradas, la técnica empleada fue la entrevista. Cada 
respuesta correcta se le asignó 2 puntos. La escala de medición para conocimientos fue: Bueno: de 13 - 18 puntos. Regular: de 7 - 12 puntos. Deficiente: de $0-$ 6 puntos. La escala de medición para prácticas fue: Adecuada: 9- 16 puntos. Inadecuada: 0 - 8 puntos.

Los datos obtenidos fueron digitalizados en una planilla Microsoft Word Excel 2010. Los datos fueron resumidos con procedimientos de estadística descriptiva. Las variables cuantitativas fueron resumidas como medias y desviaciones estándar según su ajuste a la normalidad, así como valor máximo y mínimo. Las variables cualitativas se expresaron en frecuencia absoluta (n) y relativa (\%).

Para analizar si existe asociación entre conocimiento y prácticas sobre lactancia materna exclusiva se aplicó la prueba de Chi cuadrado. Se consideró nivel de significancia estadística un valor de $\mathrm{p}<0,05$.

\section{RESULTADOS}

Hubo una proporción casi similar entre madres casadas ( $22 \%$ ) y acompañadas (31\%), pero se halló más cantidad de primerizas solteras (46\%). Respecto al nivel académico, la mayor proporción tenía enseñanza secundaria completa seguida de universitaria y en menor número de nivel primario. Respecto a la ocupación más común correspondió al de ama de casa (se dedica al hogar) con el $57 \%$ de la muestra, seguidas por las dedicadas a oficios varios (trabaja) con el $36 \%$ y las estudiantes en un $7 \%$. La edad promedio de las

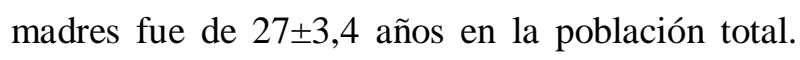
Además, se encontraron que las edades de las madres primíparas de nuestra población rondaban

Tabla 1. Características sociodemográficas las madres.

\begin{tabular}{|c|c|c|}
\hline Estado Civil & $n$ & $\%$ \\
\hline Casada & 20 & 22 \\
\hline Soltera & 41 & 46 \\
\hline Acompañada & 28 & 31 \\
\hline Separada & 1 & 1 \\
\hline Total & 90 & 100 \\
\hline \multicolumn{3}{|l|}{ Nivel de escolaridad } \\
\hline Primaria & 13 & 14 \\
\hline \multicolumn{3}{|l|}{ Estado Civil } \\
\hline Secundaria & 44 & 49 \\
\hline Universitaria & 33 & 37 \\
\hline Total & 90 & 100 \\
\hline \multicolumn{3}{|l|}{ Ocupación } \\
\hline Trabaja & 33 & 36 \\
\hline Estudia & 6 & 7 \\
\hline Se dedica al hogar & 51 & 57 \\
\hline Total & 90 & 100 \\
\hline
\end{tabular}




\begin{tabular}{|c|c|c|c|}
\hline \multicolumn{4}{|l|}{ Edad } \\
\hline & 16-19 años & 20 & 22 \\
\hline & 20-24 años & 36 & 40 \\
\hline & 25-30 años & 23 & 26 \\
\hline & 31-39 años & 11 & 12 \\
\hline Total & & 90 & 100 \\
\hline
\end{tabular}

en su mayoría entre los 16 a 30 años, siendo el rango más notable el de 20 - 24 años con un $40 \%$, por lo tanto, se pudo observar que en nuestra muestra había predominio de primerizas jóvenes y en menor número primigestas con edades comprendidas entre 31-39 años como se muestra a continuación en la Tabla 1.

Los resultados obtenidos indican que el nivel de conocimiento en lactancia materna exclusiva fue regular en un $53 \%$, bueno en un $29 \%$ y deficiente $18 \%$ en las madres primíparas encuestadas. (Tabla 2).

Tabla 2. Distribución del nivel de conocimiento en lactancia materna exclusiva en madres primíparas.

\begin{tabular}{|c|c|c|}
\hline \multicolumn{2}{|l|}{ Conocimiento } & $\%$ \\
\hline Bueno & & \\
\hline & 26 & 29 \\
\hline Regular & & \\
\hline & 48 & 53 \\
\hline Deficiente & & \\
\hline & 16 & 18 \\
\hline Total & 90 & 100 \\
\hline
\end{tabular}

El nivel de práctica encontrado fue: adecuado en un $45 \%$, inadecuado $54 \%$. Entonces se observa que casi hay una similitud entre una práctica correcta e incorrecta de amamantamiento. (Tabla 3).

Tabla 3. Distribución del nivel de práctica en lactancia materna exclusiva en madres primíparas.

\begin{tabular}{rrr}
\hline $\begin{array}{l}\text { Nivel de } \\
\text { Práctica }\end{array}$ & n & \% \\
\hline Adecuado & 41 & 45 \\
Inadecuado & 49 & 54 \\
\hline & & \\
Total & $\mathbf{9 0}$ & $\mathbf{1 0 0}$ \\
\hline
\end{tabular}

Al analizar si existe asociación entre los conocimientos y prácticas sobre lactancia materna exclusiva, no hemos encontrado asociación entre estas variables $(\mathrm{p}=, 0233)$ (Tabla 4$)$.

Tabla 4. Asociación entre conocimiento y prácticas sobre lactancia materna exclusiva*.

\begin{tabular}{ccccc}
\hline & & \multicolumn{2}{c}{$\begin{array}{c}\text { Prácticas sobre lactancia materna } \\
\text { exclusiva }\end{array}$} & \multirow{2}{*}{ Total n } \\
\cline { 3 - 4 } & & Adecuada & Inadecuada & \\
\hline \multirow{2}{*}{$\begin{array}{c}\text { Conocimiento sobre lactancia } \\
\text { materna exclusiva }\end{array}$} & Regular & 23 & 14 & 26 \\
& Deficiente & 4 & 26 & 49 \\
\hline Total & & 41 & 11 & 15 \\
\hline
\end{tabular}

* Prueba de Chi Cuadrado; $\mathrm{p}=0,233$. 


\section{DISCUSIÓN}

En el presente estudio, el nivel de escolaridad de las madres fue en su mayoría secundario, seguida del nivel universitario. El nivel de conocimiento en lactancia materna exclusiva de las madres con mayor grado de escolaridad es mejor según estudios observacionales como el de Gutiérrez Gutiérrez y colaboradores quienes indican que existe una proporción favorable entre mayor nivel de escolaridad y el grado de conocimiento de las madres acerca de las ventajas de la lactancia materna (Gutiérrez Gutiérrez et al., 2001). Sin embargo, el mantenimiento de la lactancia materna exclusiva hasta los 6 meses no tiene relación con el grado de escolaridad como indica el estudio de Pino y colaboradores, en el cual cita que inclusive las madres con grado universitario abandonan más rápido la lactancia que las madres con nivel de educación media (Pino et al., 2013). Esto podría deberse al mayor grado de responsabilidad que poseen las madres más instruidas, ya que ocupan todo su tiempo en sus respectivas actividades (Gutiérrez Gutiérrez et al., 2001).

En cuanto a la edad también se observaron primíparas más jóvenes (62 \%). En este sentido, Barriuso Lapresa, en su estudio hecho en España en el año 2007, indicaron no encontrar relación entre la edad de la madre con la prevalencia en la lactancia materna (Barriuso Lapresa, 2007). Sin embargo, Rosa y colaboradores en su artículo llamado Factores asociados a la lactancia materna exclusiva dicen que si existe relación entre la lactancia materna exclusiva con la edad materna (Rosa, et al., 2012). Esto supone que mientras más jóvenes las madres, este factor podría influir en la práctica de la lactancia materna exclusiva.

La mayoría de las madres encuestadas dijeron ser solteras (46\%). Esto hace suponer que reciben poco apoyo familiar por no tener pareja, lo cual es muy importante para continuar con la lactancia materna exclusiva y sus dificultades, como indica el estudio de Belintxon-Martín y colaboradores, realizado en España donde se encontró que las madres primerizas quedaban sorprendidas por el nivel de compromiso que lleva realizar la lactancia materna exclusiva (Belintxon-Martín et al., 2011).

Por otro lado, constatamos que el $53 \%$ de las madres primíparas en estudio tienen un nivel de conocimiento regular sobre lactancia materna exclusiva, lo que contrasta con otro estudio realizado en Perú por Huamayalli \& Solís (2014) a primigestas de las cuales el $79 \%$ desconocían sobre lactancia materna exclusiva. En otro estudio sobre conocimiento de lactancia materna exclusiva realizado por Zimmermann y colaboradores en Argentina, se encontró mayor nivel de conocimiento deficiente en las primíparas que en multíparas (Zimmermann et al., 2011).

Se pudo observar que casi hay una similitud entre una práctica correcta e incorrecta de amamantamiento (Adecuada $45 \%$ e Inadecuada $54 \%$ ), resultados que nos hace pensar que se debería disminuir la proporción de prácticas inadecuadas para poder conseguir una lactancia satisfactoria y feliz, ya que una buena práctica previene problemas de pezones enrojecidos por mal agarre del bebe, dificultad en la bajada de la leche, mala postura de amamantamiento, que se traduce en dolores de espalda o incomodidad del bebé, entre otros (Sanabria et al., 2005). 
No se encontró asociación entre las variables de conocimiento y prácticas sobre lactancia materna exclusiva, lo cual nos hace pensar que independientemente del conocimiento que puedan tener las primíparas, las prácticas pueden resultar en adecuadas o inadecuadas y que por esta razón se debe enfatizar en cuanto a la educación sobre el conocimiento de manera a que esto se pueda reflejar en prácticas adecuadas de lactancia materna exclusiva.

La mayoría de las madres primíparas presentaron un nivel de conocimiento regular sobre lactancia materna exclusiva, así como un nivel de práctica inadecuada en lactancia materna exclusiva.

No se encontró asociación entre el conocimiento y prácticas sobre lactancia materna exclusiva. Para futuras investigaciones se recomienda realizar intervenciones de educación sobre lactancia materna exclusiva a primíparas y hacer el seguimiento correspondiente para evaluar el impacto de estas sobre las prácticas en el transcurso de los primeros seis meses.

Declaración de interés. Los autores declaran no tener conflicto de intereses.

\section{REFERENCIAS BIBLIOGRÁFICAS}

Barriuso Lapresa, L. (2007). Estudio multivariante sobre la prevalencia de la lactancia materna en el centro-norte de España. Revista de Pediatría de Atención Primaria, 9, 589-612.http://archivos.pap.es/files/1116-710pdf/739.pdf

Belintxon-Martín, M., Zaragüeta, M. C., Adrián, M. C. \& LópezDicastillo, O. (2011). El comienzo de la lactancia: experiencias de madres primerizas. Anales del Sistema Sanitario de Navarra, 34(3), 409-418. http://scielo.isciii.es/scielo.php?script=sci_arttext\&pid= $\underline{\text { S1137-66272011000300007\&lng=es. }}$

Carranza, E. (2016). Conocimientos y prácticas de lactancia materna exclusiva en madres primíparas que acudieron al centro de salud Coishco Áncash, 2012. Crescendo
Ciencias de la salud., 2(2), 22-30. http://revistas.uladech.edu.pe/index.php/increscendosalud/article/view/1029/780

Centro Paraguayo de Estudios de Población (CEPEP). (2005). En Encuesta Nacional de Demografía y salud sexual y reproductiva 2004. ENDSSR 2004 (pp. 227). CEPEP. https://www.healthresearchweb.org/files/ENDSSR2004 parte_I.pdf

Gutiérrez-Gutiérrez, E., Barbosa Rodríguez, D., González Lima, R. \& Martínez Figueroa, O. (2001). Nivel de conocimiento sobre la lactancia materna. Revista Cubana de Enfermería, 17(1), 42-46.

http://scielo.sld.cu/scielo.php?script=sci_arttext\&pid=S0 864-03192001000100007\&lng=es.

Huamayalli Flores, M. \& Solís Villanueva, A. (2014). Conocimiento sobre lactancia materna exclusiva en primigestas del puesto de salud san juan. Chimbote. Crescendo Ciencias de la salud, l(2). http://revistas.uladech.edu.pe/index.php/increscendosalud/article/view/379

Ilabaca, M. J. \& Atalah, S. E. (2002). Tendencia de la lactancia materna en el Servicio de Salud Metropolitano Sur. Revista Chilena de Pediatría, 73(2), 127-134. http://www.revistachilenadepediatria.cl/index.php/rchpe $\mathrm{d} /$ article/view/1850

Instituto Nacional de Alimentación y Nutrición. (2020). Guías Alimentarias del Paraguay para Niñas y Niños Menores de 2 años. Asunción. INAM. MSPYBS. https://drive.google.com/file/d/1xQzjhotv2llX94xQYOt03N9cQZQ9c6k/view

Organización Mundial de la Salud. (2004). Estrategia Mundial: la lactancia materna, fundamental para la supervivencia infantil. Nueva York.

http://www.who.int/mediacentre/news/releases/2004/pr1 9/es/

Pino, V. J. L., López, E. M. A., Medel, I. A. P. \& Ortega, S.A. (2013). Factores que inciden en la duración de la lactancia materna exclusiva en una comunidad rural de Chile. Revista chilena de nutrición, 40(1), 48-54. https://scielo.conicyt.cl/scielo.php?script=sci arttext\&pi $\mathrm{d}=\mathrm{S} 0717-75182013000100008 \& \operatorname{lng}=\mathrm{es}$.

Rosa, N., Silva, G. \& Atalah, S. (2012). Factores asociados a la lactancia materna exclusiva. Revista chilena de pediatría, 83(2), 161-169.

http://www.scielo.cl/scielo.php?script=sci arttext\&pid= S0370-41062012000200007\&lng=es

Sanabria, M., Coronel, J., Díaz, C., Salinas, C. \& Sartori, J. (2005). Perfil de la lactancia materna en cuatro servicios de referencia neonatal. Revista Chilena de Pediatría, 76(5), 530-535.

https://scielo.conicyt.cl/scielo.php?script=sci_arttext\&pi $\mathrm{d}=\mathrm{S} 0370-41062005000500013 \& \operatorname{lng}=\mathrm{es}$.

Vásquez Munive, M., Romero Cárdenas, A. \& Rivas Castro, A. (2012). Motivaciones de la madre para amamantar: una experiencia a partir de la capacidad de escuchar. Duazary., 9(2), 116-122.

https://doi.org/10.21676/2389783X.172

Zimmermann, R., Medina Pinto, S., Ortiz Gavilán, A. V. \& Miño, C. (2011). Conocimiento sobre lactancia materna en puérperas de la ciudad de resistencia. Revista de posgrado de la VIa catedra de medicina., 207, 1-5. https://med.unne.edu.ar/sitio/multimedia/imagenes/ckfin der/files/files/revista/posgrado\%20medicina\%20VI/207. pdf 